\title{
TÄTIGKEITSBERICHT
}

\section{Die Kommission für Rechtsgeschichte Österreichs im Jahr 2010}

An dieser Stelle soll künftig ein Bericht über die Tätigkeit der Trägerorganisation dieser Zeitschrift im jeweils vergangenen Jahr erfolgen. Diesem ersten Bericht seien einige allgemeine Bemerkungen vorangestellt.

Die Kommission für Rechtsgeschichte Österreichs (KRGÖ) der Österreichischen Akademie der Wissenschaften (ÖAW) entstand 1995 durch Zusammenlegung zweier älterer Kommissionen, die auf das Jahr 1864 zurückgehen. Für die Funktionsperiode 2008-2013 wurden folgende Personen zu (ehrenamtlich tätigen) Mitgliedern der KRGÖ gewählt: Hon.-Prof. HR Dr. Leopold Auer (Wien), w.M. Univ.-Prof. i.R. Dr. Ernst Bruckmüller (Wien, 2009 ausgeschieden), o. Univ.-Prof. Dr. Ursula Floßmann (Linz) o. Univ.-Prof. DDr. Herbert Kalb (Linz), emer. o. Univ.-Prof. Dr. Dr.h.c. Gernot Kocher (Graz), ao. Univ.-Prof. Dr. Christian Neschwara (Wien), w.M. emer. o. Univ.-Prof. Dr. DDr.h.c. Werner Ogris (Wien), k.M. ao. Univ.-Prof. Dr. Thomas Olechowski (Wien), ao. Univ.-Prof. Dr. Ilse ReiterZatloukal (Wien), k.M. emer. o. Univ.-Prof. Dr. Dr.h.c. Gerhard Thür (Wien), emer. o. Univ.-Prof. Dr. Gunter Wesener (Graz), w.M. Univ.-Prof. Dr. Dr.h.c. Thomas Winkelbauer (Wien). Am 16. Juni 2010 legte Ogris nach 35 Jahren Obmannschaft sein Amt zurück; zu seinem Nachfolger wurde Olechowski gewählt. Zugleich wurde Ogris als Nachfolger von Kocher zum Obmann-Stellvertreter gewählt. Anstelle des ausgeschiedenen Kommissionsmitglieds Bruckmüller wurde in derselben Sitzung w.M. emer.o. Univ.-Prof.
Dr. Herbert Matis zum neuen Kommissionsmitglied gewählt.

Bis 2003 besaß die KRGÖ weder eigene Mitarbeiter noch Räume, sondern wurde vom Obmann neben seiner Funktion als Ordinarius an der Universität Wien geleitet. Erst im Gefolge von Ogris' Emeritierung wurde die KRGÖ parallel zur beständigen Ausweitung ihrer Tätigkeit - mit Personal und Räumen ausgestattet; seit 2008 verfügt sie über zweieinhalb Planstellen und hat ihren Sitz seit 2007 in Wien III., Strohgasse 45. Wissenschaftliche Mitarbeiterinnen im Jahr 2010 waren Dr. Eva Ortlieb und MMag. Kathrin Kininger, welch letztere im Mai ausschied, worauf ihre Planstelle geteilt und je zur Hälfte mit Mag. Kamila Staudigl-Ciechowicz und DDr. Christoph Schmetterer nachbesetzt wurde. Die Administration wurde von der halbtägig angestellten Katharina Bernold besorgt. Dazu kam eine vom Wissenschaftsfonds FWF finanzierte Mitarbeiterin, Mag. Ellen Franke; im Rahmen des Kooperationsprojekts mit der Akademie der Wissenschaften zu Göttingen (siehe sogleich) waren Mag. Thomas Schreiber und Mag. Sandra Weiss auf Rechnung der Göttinger Akademie halbtägig bei der KRGÖ angestellt.

Für das Jahr 2010 wurde die KRGÖ von der ÖAW mit einem Budget von $€ 120.500$ ausgestattet; dazu kamen $€ 39.584$ von der Göttinger Akademie, $€ 33.136$ vom FWF und $€ 8.200$ von sonstigen Drittmittelgebern. Der Drittmittelanteil betrug somit $40,2 \%$. 
Von ihren Vorgängerinstitutionen übernahm die KRGÖ die Aufgabe, Quellen zur österreichischen Rechtsgeschichte zu edieren, und zwar in der Reihe „Fontes iuris", einer Unterreihe der „Fontes Rerum Austriacarum“. Am 1. Oktober 2010 konnte im Ungarischen Kulturinstitut in Bratislava der Band 21/1 der „Fontes iuris“ präsentiert werden: „Das Preßburger Protocollum Testamentorum 1417 (1427) - 1529, Teil 1: 14101487, hgg. v. Dr. Judit Majorossy und Univ.-Prof. Dr. Katalin Szende. Es handelt sich um eine für Ostmitteleuropa einzigartig dichte Quellenüberlieferung: Das Preßburger Protocollum umfasst etwa ein Drittel aller aus dem mittelalterlichen Ungarn erhalten gebliebene letztwilligen Verfügungen und etwa $95 \%$ der Testamente aus dem mittelalterlichen Preßburg. Der zweite Teil, der die Einträge von 1487 bis 1529 enthalten wird, ist derzeit in Vorbereitung. 2010 fertig gestellt wurde ferner eine von Kommissionsmitglied Neschwara besorgte Edition der „ältesten Materialien zum ABGB“, die nach interner Begutachtung in der KRGÖ im Jänner 2011 bei der Publikationskommission der ÖAW eingereicht wurde. Im Berichtsjahr 2010 wurde ferner insbesondere an einer Fortsetzung der Edition der Wiener Stadtbücher (von denen bislang vier Bände im Rahmen der „Fontes Iuris“ erschienen sind; Bearbeiter des fünften Bandes sind Univ.-Prof. Dr. Gerhard Jaritz und Neschwara), an Rechtsquellen der Stadt Tulln (Bearbeiter: Mag. Johannes Ramharter), sowie an Relationen des Reichshofrates Georg Adam von Starhemberg (Bearbeiterin: Ortlieb) gearbeitet.

Seit 2004 sind Forschungen zum Reichshofrat (RHR) ein besonderer Forschungsschwerpunkt der KRGÖ. Der RHR war vom 16. bis zum Anfang des 19. Jahrhunderts eines der Höchstgerichte des Heiligen Römischen Reiches, kaiserliche Behörde insbesondere bei Lehens- und Privilegienangelegenheiten sowie kaiserliches Ratsgremium. Seine Akten befinden sich zum größten Teil im Haus-, Hof- und Staatsarchiv in Wien. Ihre Erschließung erfolgt seit 2007 im
Rahmen eines Langzeitprojekts der Akademie der Wissenschaften zu Göttingen in Kooperation mit dem Archiv und der KRGÖ. Daneben betreibt die KRGÖ eigene Forschungsprojekte in Bezug auf den RHR. Außer den vorhin erwähnten Starhemberg-Relationen ist hier insbesondere das Projekt "Appellationen an den Reichshof-

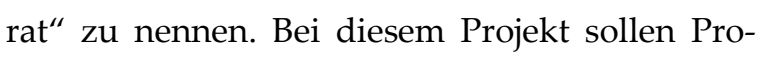
zessakten des RHR aus dem für die Durchsetzung der Appellation entscheidenden Zeitraum zwischen 1519 und 1740 herangezogen und ausgewertet werden, wobei sowohl juristische als auch geschichtswissenschaftliche Fragen an die Quellen gestellt werden, so etwa nach Prozessrecht und Rechtsanwendung, aber auch nach der Rolle von Reichsoberhaupt und Reichsgericht in der Rechtswirklichkeit des territorial strukturierten Reichs. Das von Kommissionsmitglied Auer geleitete Projekt wird vom FWF finanziert (Projektnummer P 20586; Projektzeitraum: 2008-2013). Bearbeiterin des Projekts ist Franke. Neben mehreren Aufsätzen und Vorträgen waren im Berichtsjahr 2010 insbesondere eine Tagung für 2011 und eine Monographie für 2013 in Vorbereitung.

Von den sonstigen Projekten der KRGÖ im Berichtszeitraum ist hier noch die Festschrift für Werner Ogris, „Grundlagen der österreichischen Rechtskultur", zu nennen, die von den Kommissionsmitgliedern Olechowski und Neschwara gemeinsam mit ao.Univ.-Prof. Dr. Alina Lengauer herausgegeben und am 27. Oktober 2010 präsentiert wurde; mehrere Mitglieder und Mitarbeiter/innen der KRGÖ haben mit Aufsätzen zu dieser Festschrift beigetragen.

Thomas Olechowski 
